\title{
Criptografía Visual Basada en el Esquema de Umbral: Una Revisión Tutorial
}

\author{
Mariko Nakano $^{\left({ }^{* 1}\right)}$, Enrique Escamilla ${ }^{(1)}$, Héctor Pérez ${ }^{(1)}$, Mitsugu Iwamoto ${ }^{(2)}$ \\ (1) Escuela Superior de Ingeniería Mecánica y Eléctrica, Instituto Politécnico Nacional, \\ Av. Santa Ana 1000, Col. San Francisco Culhuacan, 04430 México D. F.-México. \\ (e-mail: mnakano@ipn.mx, eescamillah@ipn.mx, hmperezm@ipn.mx) \\ (2) Departamento de Ingeniería en Informática y Comunicaciones, Universidad de Electro- \\ Comunicaciones, 1-5-1 Chofugaoka, Chofu, Tokio 182-8585, Japón. \\ (e-mail: mitsugu@quest.is.uec.ac.jp)
}

Recibido Dic. 10, 2010; Aceptado Feb. 08, 2011; Versión Final Recibida Mar. 21, 2011

\section{Resumen}

En este artículo se realiza una revisión sobre criptografía visual (CV), una representación visual del esquema de secreto compartido y que está llamando poderosamente la atención debido a sus potenciales aplicaciones en el sistema de banca electrónica, así como en sistemas de identificación personal. En la CV, una imagen binaria, tal como letras, logotipos o imágenes, se comparte entre los distintos participantes mediante un conjunto de imágenes binarias llamadas "sombras". Hasta la fecha han sido propuestos varios esquemas de CV, tales como la CV basada en el esquema de umbral, la CV de acceso general y la CV extendida. Se analizan también las condiciones que se deben cumplir para la recuperación del mensaje secreto, así como los aspectos de seguridad más relevantes de este esquema.

Palabras clave. criptografía visual, esquema de secreto compartido, esquema de umbral

\section{Threshold Based Visual Cryptography: A Tutorial Review}

\begin{abstract}
In this paper, a review for threshold-based visual cryptography (VC), a visual representation of the secret sharing scheme is presented. The VC has potential applications in the electronic banking system, as well as in personal identification systems. En la VC a binary image such as letters, logotypes or halftone images are shared among a group of participants using a set of images, so called shares. Until now several VC schemes, such as threshold-based VC, the general access VC and the extended VC, have been proposed. The conditions that must be satisfied for a correct recovery of the secret message, as well as the most relevant security issues of this scheme are also analyzed.
\end{abstract}

Keywords. visual cryptography, secret sharing scheme, threshold based scheme 


\section{INTRODUCCIÓN}

La criptografía visual (CV) fue propuesta por Naor y Shamir (1994), como una representación visual del esquema de secreto compartido (ESC) (Shamir 1979). En el ESC una llave numérica es considerada como un secreto, el cual se comparte entre todos los participantes (Shamir 1979), mientras que en $\mathrm{CV}$ el secreto es una imagen binaria la cual se comparte de manera similar entre todos los participantes. La Fig. 1 muestra tres ejemplos de secretos visuales que pueden ser usados en un esquema de CV. Como se puede ver de la Fig. 1 las letras representadas por una imagen binaria, un logotipo binario o una imagen generada usando la técnica conocida como "halftone" descrita en Mese y Vaidyanathan (2002), se pueden usar como secretos visuales.

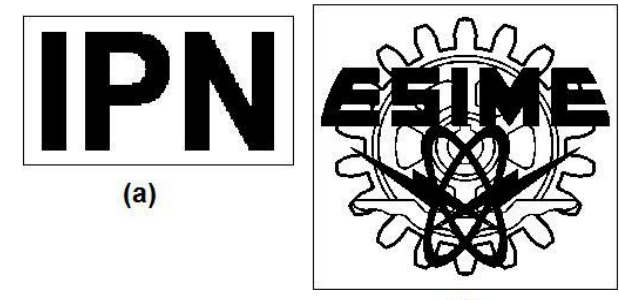

(b)

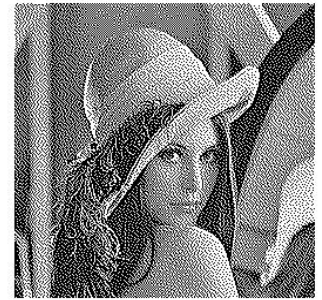

(c)

Fig. 1: Ejemplos de secretos visuales. (a) Letras representadas por imagen binaria, (b) logotipo binario, (c) imagen binaria usando técnica de "halftone".

En la CV, cada participante recibe una imagen binaria llamada sombra, generada en forma tal que ningún participante es capaz de revelar la imagen secreta, usando únicamente su propia sombra, siendo necesario que los participantes calificados para ello sobrepongan sus sombras con el fin de que la imagen secreta puede ser revelada. La diferencia principal entre la CV y el esquema de secreto compartido convencional (Shamir 1979) es que en la CV, no se requiere ningún conocimiento previo acerca del algoritmo criptográfico empleado, tal como sucede con la llave secreta, ni poder computacional para revelar el secreto. En la CV el proceso de revelación del secreto compartido se basa en la operación de superposición o "stacking" de las sombras, a partir de la cual el sistema visual humano (SVH) percibe la imagen secreta. Esta operación se puede realizar tanto digitalmente como analógicamente, en cuyo caso las sombras son acetatos con las imágenes de las sombras impresas en ellos. En este caso la operación requerida consiste realmente en superponer un acetato con otro físicamente. Por su parte, en el caso de las sombras digitales, la realización digital de la operación de superposición es equivalente a una operación "OR" bit a bit.

La CV convencional propuesta por Naor y Shamir se conoce también como CV basado en el esquema de umbral y se denota como $(k, n)$-CV (Naor y Shamir 1994). Esta notación significa que para revelar el secreto visual se requieren cuando menos $k$ de todos los $n$ participantes. Es decir que cualquier número de participantes mayor o igual a $k$ pueden revelar el secreto; sin embargo cualquier número menor, por ejemplo $(k-1)$, no pueden lograrlo. Después de la propuesta de Naor y Shamir (1994), aparecieron algunas variantes de la CV, siendo las más importantes los esquemas de acceso general de CV (Ateniese et al. 1996) y el esquema extendido de CV (Ateniese et al. 1997, Droste 1996, Klein y Wessler 2007).

Recientemente los esquemas de CV están llamando poderosamente la atención por sus posibles aplicaciones, tales como el sistema de banca por internet, banca electrónica o "Internet Banking" (Hegde et al. 2008), archivos multimedia (Mora y Torrabia 2002), así como sistemas de identificación personal combinando la CV con las técnicas de biométricas (Rao et al. 2008), etc.

En este artículo se realiza una revisión tutorial sobre CV basado en el esquema de umbral propuesto por Naor y Shamir (1994), se describen básicamente los esquemas $(2, n),(3, n)$ y $(n, n)$, junto con algunos ejemplos. Se analizan además las condiciones que se deben cumplir para la recuperación del mensaje secreto, así como los aspectos de seguridad más relevantes de este esquema. Los programas computacionales están disponibles en sección "enlaces" de la página www.posgrados.esimecu.ipn.mx/. 
El resto de este artículo está compuesto de la siguiente manera: en la Sección "Principios básicos de la CV", se describen conceptos que se manejan en todos los esquemas de CV incluyendo el esquema de umbral $(k, n)$-CV, los cuales son sombras, los algoritmos de cifrado y descifrado, y las condiciones de contraste y seguridad en una forma general. En la Sección de "Esquema $(k, n)-C V$ ", se describen las formulas que se usan para generar las matrices básicas en $(2, n)-\mathrm{CV},(3, n)$-CV y $(n, n)-\mathrm{CV}$, junto con algunos ejemplos. Finalmente se concluye el artículo en "Conclusiones".

\section{PRINCIPIOS BÁSICOS DE LA CV}

\section{Sombras}

En cualquier algoritmo de criptografía visual (CV), se generan imágenes binarias llamadas sombras, de las cuales cada participante recibe una sombra específica. Las sombras pueden ser ya sea imágenes de ruido aleatorio, cuando se aplica el esquema de $(k, n)$-CV (Naor y Shamir, 1994) o el algoritmo de acceso general (Ateniese et al. 1996), como se muestra en la Fig. 2 (a), o pueden ser imágenes visualmente reconocibles cuando el algoritmo de CV extendido (Ateniese et al. 1997, Droste 1996, Klein y Wessler 2007) es usado, como se muestra en la Fig. 2 (b).

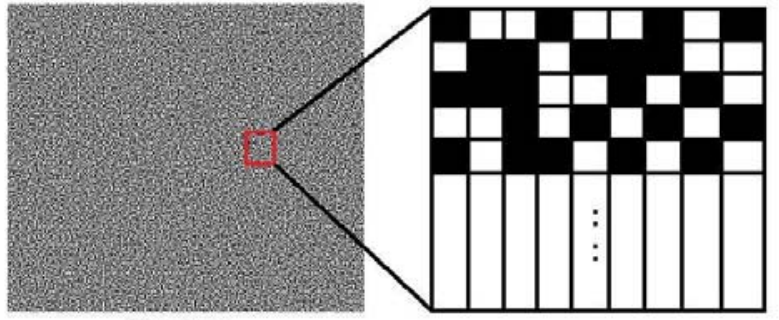

Sombra

(a)

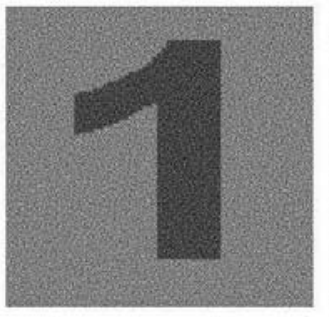

Sombra 1

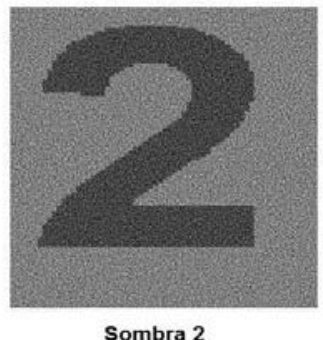

(b)

Fig. 2: Ejemplos de sombras. (a) sombra con apariencia de ruido aleatorio, (b) sombras visualmente reconocible

Obviamente las sombras visualmente reconocibles proporcionan un mayor número de ventajas, que aquellas que no lo son (Naor y Shamir 1994, Ateniese et al. 1996), debido a que usando sombras visualmente reconocibles se puede distinguir a un participante de otro. Sin embargo el algoritmo de construcción de la $\mathrm{CV}$ en este caso es más complejo y generalmente el tamaño de la sombra se expande más respecto al tamaño de la imagen secreta.

Algoritmo genérico de cifrado en $\mathrm{CV}$

En CV, la imagen secreta denotada como IS se cifra en varias sombras para compartir entre los participantes, por lo tanto la generación de sombras es equivalente a la operación de cifrado. Las sombras se generan usando matrices llamadas matrices básicas. La variación entre los algoritmos de CV, tales como los $(k, n)-C V$, los de acceso general CV y los de CV extendida, está en el método de generación de las matrices básicas que se usan para la construcción de las sombras de cada uno de los participantes. Sin embargo una vez que las matrices se han generado, el proceso de construcción de las sombras es igual para todas las variantes. Por lo tanto aquí solo se explica el proceso de construcción de sombras usando las matrices básicas determinadas de antemano. Las matrices básicas tienen una dimensión de $n$ renglones y $m$ columnas como se muestra en (1), donde $n$ es el número de participantes y $m$ es el factor de expansión de cada pixel de la imagen secreta.

$$
S^{0}=\left[\begin{array}{cccc}
s_{1,1}^{0} & s_{1,2}^{0} & \ldots & s_{1, m}^{0} \\
s_{2,1}^{0} & s_{2,2}^{0} & \ldots & s_{2, m}^{0} \\
\cdot & \cdot & \ldots & \cdot \\
\cdot & \cdot & \ldots & \cdot \\
s_{n, 1}^{0} & s_{n, 2}^{0} & \ldots & s_{n, m}^{0}
\end{array}\right], S^{1}=\left[\begin{array}{cccc}
s_{1,1}^{1} & s_{1,2}^{1} & \ldots & s_{1, m}^{1} \\
s_{2,1}^{1} & s_{2,2}^{1} & \ldots & s_{2, m}^{1} \\
\cdot & \cdot & \ldots & \cdot \\
\cdot & \cdot & \ldots & \cdot \\
s_{n, 1}^{1} & s_{n, 2}^{1} & \ldots & s_{n, m}^{1}
\end{array}\right]
$$


Aquí, la matriz $S^{0}$ se usa cuando el pixel de la imagen secreta IS es blanco, mientras que cuando el pixel de la imagen secreta $I S$ es negro se usa la matriz $S^{1}$, donde en ambos casos los elementos $s_{i, j}^{b}$ son binarios, esto es $s_{i, j}^{b} \in\{0,1\}, b \in\{0,1\}$. La Fig. 3 muestra el algoritmo genérico de cifrado en $\mathrm{CV}$.

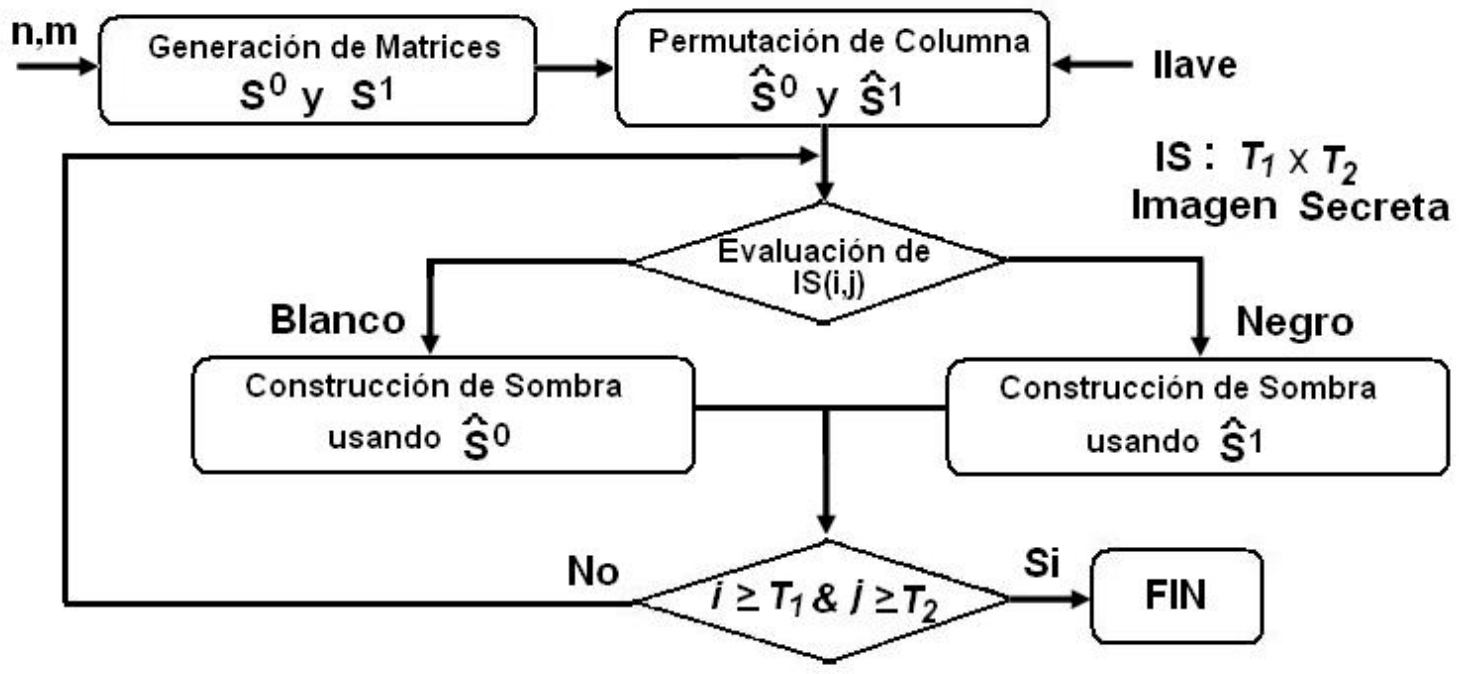

Fig. 3 Diagrama bloque del algoritmo genérico de cifrado en CV.

Las matrices básicas generadas de acuerdo al esquema de CV empleado, se permutan por columna con una llave, aunque ésta no se necesita en el proceso de revelación del secreto. El número total de permutaciones existentes es de $m$ !. Un ejemplo de la operación de permutación cuando $m=4$ con un vector de permutación $v p=[2,1,4,3]$, se muestra a continuación.

$S^{b}=\left[\begin{array}{cccc}s_{1,1}^{b} & s_{1,2}^{b} & s_{1,3}^{b} & s_{1,4}^{b} \\ s_{2,1}^{b} & s_{2,2}^{b} & s_{2,3}^{b} & s_{2,4}^{b} \\ \vdots & \vdots & \vdots & \vdots \\ s_{n, 1}^{b} & s_{n, 2}^{b} & s_{n, 3}^{b} & s_{n, 4}^{b}\end{array}\right] \stackrel{V p}{\longrightarrow} \hat{S}^{b}=\left[\begin{array}{cccc}s_{1,2}^{b} & s_{1,1}^{b} & s_{1,4}^{b} & s_{1,3}^{b} \\ s_{2,2}^{b} & s_{2,1}^{b} & s_{2,4}^{b} & s_{2,3}^{b} \\ \vdots & \vdots & \vdots & \vdots \\ s_{n, 2}^{b} & s_{n, 1}^{b} & s_{n, 4}^{b} & s_{n, 3}^{b}\end{array}\right]$

donde $b \in\{0,1\}$. Se puede usar el mismo vector de permutación para generar las dos matrices básicas, o éstos pueden ser diferentes, dependiendo de la aplicación en particular. Los subpixeles de la sombra del i-ésimo participante corresponden al i-ésimo renglón de la matriz $\hat{S}^{0} \circ \hat{S}^{1}$, dependiendo del valor del pixel de la imagen secreta. Por ejemplo si $n=2$ y $m=4$, las matrices básicas $S^{0}$ y $S^{1}$ están dado por

$S^{0}=\left[\begin{array}{llll}1 & 1 & 0 & 0 \\ 1 & 1 & 0 & 0\end{array}\right], \quad S^{1}=\left[\begin{array}{llll}1 & 1 & 0 & 0 \\ 0 & 0 & 1 & 1\end{array}\right]$

Seguidamente permutando las columnas usando el vector de permutación $v_{p}=[2,4,3,1]$, obtenemos

$\hat{S}^{0}=\left[\begin{array}{llll}1 & 0 & 0 & 1 \\ 1 & 0 & 0 & 1\end{array}\right], \quad \hat{S}^{1}=\left[\begin{array}{llll}1 & 0 & 0 & 1 \\ 0 & 1 & 1 & 0\end{array}\right]$

Dependiendo del color (blanco o negro) del pixel de la imagen secreta, se selecciona una de las dos matrices permutadas $\hat{S}^{0} \circ \hat{S}^{1}$. Si un pixel de la imagen secreta $I S(i, j)=0$ (blanco), la matriz 
$\hat{S}^{0}$ se emplea para la generación de las sombras, en otro caso $I S(i, j)=1$ (negro), la matriz $\hat{S}^{1}$ se emplea para la generación de las mismas. La Fig. 4 muestra un ejemplo de generación de dos sombras (Fig. 4(b) y (c)) dando la imagen secreta mostrada la Fig. 4(a). Aquí las matrices básicas usadas están dadas por (3) con diferentes permutaciones. Las permutaciones que se aplicaron para cada pixel de la imagen secreta son $v_{p}=[1,2,3,4], v_{p}=[4,1,2,3], v_{p}=[2,4,1,3], v_{p}=[2,3,4,1]$, respectivamente. El primer pixel de la imagen secreta $I S(1,1)=1$ (negro), la matriz $\hat{S}^{1}$ se emplea para la generación de las sombras, siendo la sombra del primer participante aquella generada usando el primer renglón $V_{1}=\left[\begin{array}{llll}1 & 1 & 0 & 0\end{array}\right]$ de la matriz $\hat{S}^{1}$; mientras que la sombra del segundo participante se genera usando el segundo renglón, $V_{2}=\left[\begin{array}{llll}0 & 0 & 1 & 1\end{array}\right]$, de matriz $\hat{S}^{1}$. Así, para mantener el radio de aspecto de la imagen revelada respecto a la imagen secreta original, los vectores $V_{1}, V_{2}$ se convierten en matrices de $2 \times 2$ como se muestra en (5).

$V_{1}=\left[\begin{array}{llll}1 & 1 & 0 & 0\end{array}\right] \Rightarrow\left[\begin{array}{ll}1 & 1 \\ 0 & 0\end{array}\right], \quad V_{2}=\left[\begin{array}{llll}0 & 0 & 1 & 1\end{array}\right] \Rightarrow\left[\begin{array}{ll}0 & 0 \\ 1 & 1\end{array}\right]$

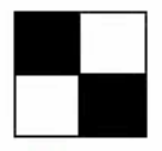

(a)

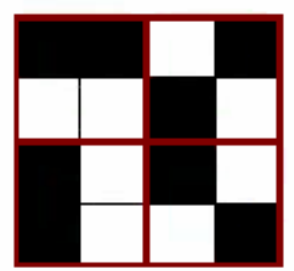

(b)

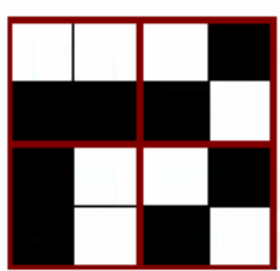

(c)

Fig. 4 Concepto de sub-pixeles y expansión de pixeles $(m=4)$. (a) 4 pixeles de una imagen secreta, (b) posible sombra 1 y (c) posible sombra 2.

\section{Operación de descifrado en CV}

Para revelar el secreto, esto es descifrar la CV, los participantes calificados para ello tienen que superponer sus sombras, sin importar el orden en que ellos lo hagan. Como se mencionó anteriormente, la operación de superponer información visual es una operación lógica-OR en su realización digital, mientras que la realización analógica, consiste en superponer físicamente entre sí, uno o más acetatos conteniendo las sombras. La operación lógica-OR se muestra en la Fig. 5. Como se puede observar de esta figura, el resultado de la operación lógica-OR, aunque es también binario, aumenta el número de pixeles en las sombras de cada participante en comparación con el tamaño de la imagen original.

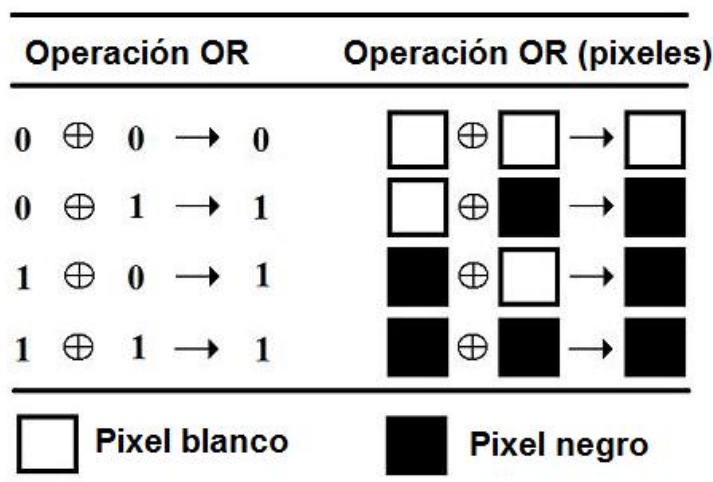

Fig. 5 Operación lógica-OR 
La Fig. 6 muestra el resultado de la superposición de las dos sombras generadas (Fig. 4 (b) y (c)) en el ejemplo anterior.

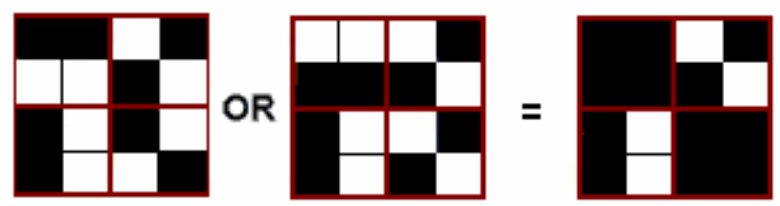

Fig. 6 Un ejemplo de superposición usando operación lógica-OR

Comparando el resultado obtenido en la Fig. 6 y la imagen secreta (Fig. 4 (a)), podemos ver que el pixel negro de la imagen secreta se expande a 4 pixeles negros, mientras que el pixel blanco de la imagen secreta se expande a 2 pixeles negros y 2 pixeles blancos en la imagen revelada. La imagen secreta observada a partir de la imagen resultante mostrada en la Fig.6 podría no ser tan clara, sin embargo el tamaño real de un pixel es muy pequeño y esto ayuda diferenciar la parte correspondiente a los pixeles negros de la parte correspondiente a los blancos como se puede percibir de la Fig. 7. La Fig. 7(a) es la imagen secreta y la Fig. 7(b) es un resultado de la superposición de las dos sombras generadas por el algoritmo genérico de cifrado de CV mostrado en la Fig. 3 con las matrices básicas dadas por (3).

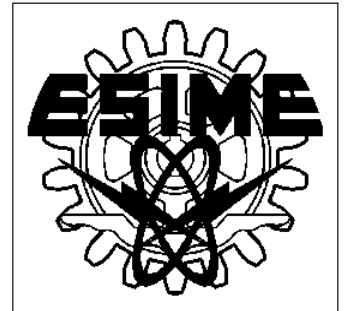

(a)

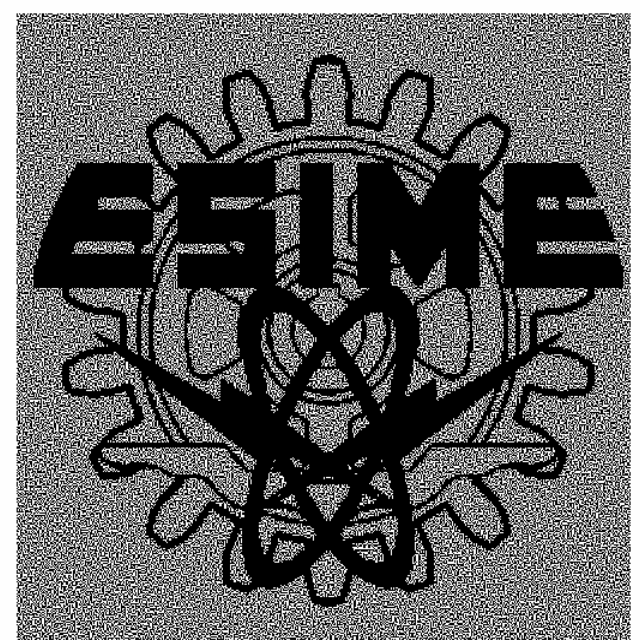

(b)

Fig. 7 La imagen secreta y la imagen revelada por la superposición de las sombras

\section{Condiciones de contraste y seguridad}

Las matrices básicas dadas por (1) deben cumplir dos condiciones para que el esquema de CV sea completo y la operación de superponer las sombras de los participantes calificados para ello, sean capaces de revelar el secreto.

La primera condición es la de contraste, la cual relaciona el contraste entre los pixeles negros y los pixeles blancos de la imagen revelada. Considerando un conjunto de participantes calificados $\left[i_{1}, i_{2}, \cdots, i_{k}\right]$, la operación lógica-OR entre los renglones correspondientes a las matrices básicas $S^{0}, S^{1}$, de los participantes calificados deben cumplir las dos condiciones siguientes

$$
\begin{aligned}
& \operatorname{Hw}\left(\operatorname{OR}\left(V_{i 1}^{0}, V_{i 2}^{0}, \cdots, V_{i k}^{0}\right)\right)<d_{v}-\alpha m, \\
& \operatorname{Hw}\left(\operatorname{OR}\left(V_{i 1}^{1}, V_{i 2}^{1}, \cdots, V_{i k}^{1}\right)\right) \geq d_{v}
\end{aligned}
$$


donde $H w(X)$ son los pesos de Hamming del vector $X$, por ejemplo $H w\left(\left[\begin{array}{llll}1 & 0 & 1 & 0\end{array}\right]\right)=2$, $H w\left(\left[\begin{array}{llll}1 & 1 & 1 & 0\end{array}\right]\right)=3$. Aquí $V_{i q}^{b}$ denota el iq-ésimo renglón de la matriz $S^{b}$, con $b \in\{0,1\}, d_{v}$ es un valor de umbral, $\alpha$ representa la diferencia relativa entre negro y blanco de las sombras superpuestas. Las condiciones dadas por (6) garantizan que el secreto visual sea perceptible por medio de la superposición de las sombras de los participantes calificados.

Para determinar las condiciones básicas de un esquema óptimo de $C V, m$ debe minimizar el incremento en el tamaño de la sombra y por lo tanto el incremento en el tamaño de la imagen revelada con respecto a la original, mientras que $\alpha$, debe maximizar el contraste de la imagen descifrada, cuyo cálculo depende del esquema en particular de CV empleado (Noar y Shamir 1997, Verhuel Van Tilborg 1997, Blundo et al. 2001, Blundo et al. 2006, Hajiabolhassan y Cheraghi 2007) .

Adicionalmente otro aspecto de gran relevancia es el que se refiere a la seguridad, en donde para cualquier conjunto de participantes prohibidos $\left[j_{1}, j_{2}, \ldots, j_{q}\right]$ se debe satisfacer la siguiente condición de seguridad.

$$
\operatorname{Hw}\left(\operatorname{OR}\left(V_{j_{1}}^{0}, \cdots, V_{j_{q}}^{0}\right)\right)=\operatorname{Hw}\left(O R\left(V_{j_{1}}^{1}, \cdots, V_{j_{q}}^{1}\right)\right)
$$

Esta condición garantiza que la operación de superponer las sombras del conjunto prohibido no pueda revelar la imagen secreta (Naor y Shamir 1994, Naor y Shamir 1997).

\section{ESQUEMA $(k, n)-C V$}

En el esquema ( $k, n)$-CV propuesto en (Naor y Shamir 1994), las $n$ sombras se generan para ser repartidas entre los $n$ participantes, en forma tal que sí al menos un número $k$ de participantes, dado de antemano, superponen sus sombras el secreto visual puede ser revelado. Sin embargo la operación de superponer cualquier número de participantes menor que $k$, por ejemplo, (k-1), no debe ser capaz de revelar el secreto. Las sombras que se manejan en este esquema son imágenes binarias que tienen una apariencia de ruido aleatorio como se muestran en la Fig. 2 (a).

En (Naor y Shamir 1994), los métodos empleados para generar las matrices básicas de los tres esquemas: $(2, n)-C V,(3, n)-C V$ y $(n, n)-C V$ fueron descritos en forma general, sin embargo no se presentan los algoritmos requeridos para generar los esquemas $(k, n)-\mathrm{CV}$, con cualquier $k \geq 2$. Además, usando el esquema de acceso general se puede generar cualquier esquema de $\mathrm{CV}$, incluyendo el $(k, n)$-CV (Koga et al. 2001). Por lo anterior en esta sub-sección se describen solamente los algoritmos de $(2, n)-\mathrm{CV},(3, n)-\mathrm{CV}$ y $(n, n)-\mathrm{CV}$.

\section{Esquema (2,n)-CV}

En este esquema, cualquier combinación de dos de los $n$ participantes pueden revelar la imagen secreta, superponiendo sus sombras. Una forma de construcción de las matrices básicas para lograr este fin es la siguiente, aunque esta no es la única forma de hacerlo. Defina las matrices básicas $S^{0}, S^{1}$ como $2 \times n$ matrices con

$$
\begin{aligned}
& S^{1}(i, j)= \begin{cases}1, & i=j \\
0, & i \neq j\end{cases} \\
& S^{0}(1,:)=S^{1}(1,:), \quad S^{0}\left(i_{k 1},:\right)=S^{0}\left(i_{k 2},:\right), i_{k 1}=1, \ldots n, i_{k 2}=1, \ldots n
\end{aligned}
$$

La diferencia óptima relativa que se puede tener en un esquema de $(2, n)$-CV está dada por (Stinson 1999) 
$\alpha=\gamma^{*}(n)=\frac{\lfloor n / 2\rfloor\lceil n / 2\rceil}{n(n-1)}$

Donde $n$ es el número de participantes. Las diferencias relativas óptimas para $n=2,3$ y 4 son

$\gamma^{*}(2)=\frac{1}{2}, \gamma^{*}(3)=\frac{1}{3}, \gamma *(4)=\frac{1}{3}$

Las matrices básicas del esquema (2,4)-CV, las cuales también se pueden obtener usando (8) y (9), están dadas por

$S^{0}=\left[\begin{array}{llll}1 & 0 & 0 & 0 \\ 1 & 0 & 0 & 0 \\ 1 & 0 & 0 & 0 \\ 1 & 0 & 0 & 0\end{array}\right] \quad, S^{1}=\left[\begin{array}{llll}1 & 0 & 0 & 0 \\ 0 & 1 & 0 & 0 \\ 0 & 0 & 1 & 0 \\ 0 & 0 & 0 & 1\end{array}\right]$

La diferencia relativa $\alpha$ de este esquema es $\alpha=1 / 4$. Sin embargo (11) indica que la diferencia relativa óptima para $(2,4)-\mathrm{CV}$ es $\alpha=1 / 3$, esto significa que existe otra realización, o sea otras matrices básicas. En realidad las matrices básicas mostradas en (13) ofrecen la diferencia relativa óptima $(\alpha=1 / 3)$, aunque el factor de expansión es $m=6$, por lo tanto la imagen revelada se distorsiona, expandiendo más ancho que alto de la imagen.

$S^{0}=\left[\begin{array}{llllll}1 & 1 & 1 & 0 & 0 & 0 \\ 1 & 1 & 1 & 0 & 0 & 0 \\ 1 & 1 & 1 & 0 & 0 & 0 \\ 1 & 1 & 1 & 0 & 0 & 0\end{array}\right], S^{1}=\left[\begin{array}{llllll}1 & 1 & 1 & 0 & 0 & 0 \\ 1 & 0 & 0 & 1 & 1 & 0 \\ 0 & 1 & 0 & 1 & 0 & 1 \\ 0 & 0 & 1 & 0 & 1 & 1\end{array}\right]$

La Fig. 8 muestra la comparación de ambos esquemas de (2,4)-CV.

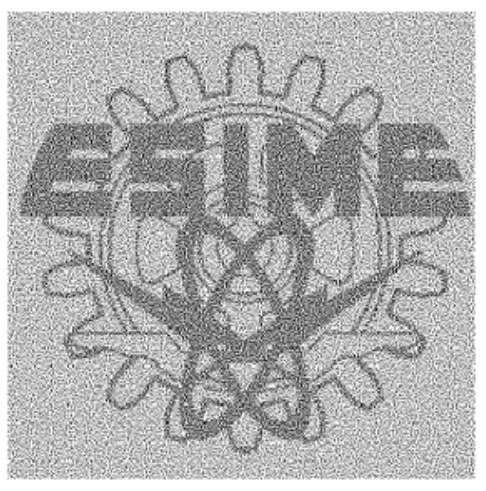

(a)

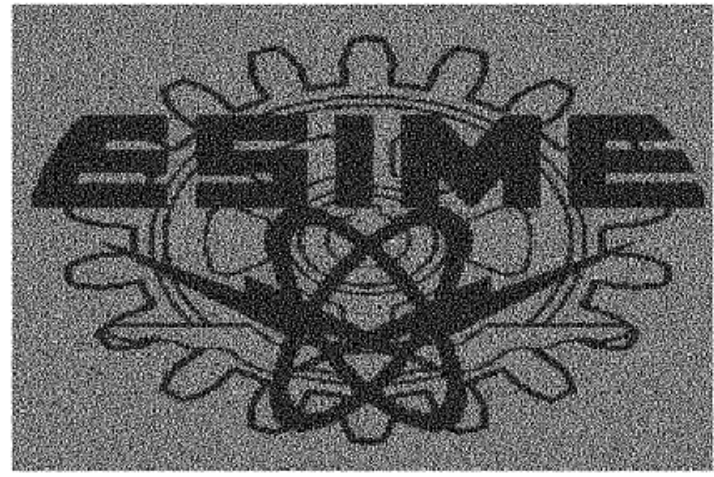

(b)

Fig. 8 Comparación de imágenes reveladas entre dos realizaciones de (2,4)-CV. (a) Realización sin deformación usando (12), (b) realización con deferencia relativa óptima usando (13).

Aquí cuando el número de participantes aumenta hacia infinito, o sea cuando $n \rightarrow \infty$, la diferencia relativa $\alpha$ está dada por (10) la cual converge a $1 / 4$ como se muestra (14).

$\alpha^{*}=\lim _{n \rightarrow \infty} \gamma^{*}(n)=\lim _{n \rightarrow \infty} \frac{\mid n / 2\rfloor \mid n / 2\rceil}{n(n-1)}=\frac{1}{4}$ 
Para mantener un $\alpha$ óptimo, probablemente hay que sacrificar el factor de expansión $m$, como se puede observar en la Fig. 8.

El asunto de la diferencia relativa óptima (contraste óptimo), el factor de expansión y la construcción de las matrices básicas para el esquema de $(2, n)$-CV fue propuesto en (Blundo et al., 1999, Naor y Shamir 1997, Stinson 1999), bajo ciertas condiciones. Primero el número de participantes $n$ tiene que ser un número primo y $n=\bmod 4$. Así las matrices básicas $S^{0}, S^{1}$ están dadas por

$$
S^{1}(1, k)=\left\{\begin{array}{ll}
1 & k \in Q \\
0 & k \notin Q
\end{array} \quad k=1, \ldots n \quad, \quad S^{1}(r,:)=\operatorname{shift}\left(S^{1}(1,:), r-1\right)\right.
$$

Donde $Q(n)$ es residual cuadrático modulo $n$ y esta dado por

$Q(n)=\left\{i^{2} \bmod n: 1 \leq i \leq(n-1) / 2\right\}$

La matriz $S^{0}$ se calcula como

$$
S^{0}(:, 1: \operatorname{num}(Q(n)))=1 \quad, \quad S^{0}(:, \text { num }(Q(n))+1: n)=0
$$

donde num $(Q(n))$ es el número de elementos de $Q(n)$. Como un ejemplo consideramos $n=11$, el cual cumple el requisito para $n$. Antes de obtener las matrices básicas, hay que obtener $Q(11)$. De acuerdo a (16), obtenemos $Q(11)=\{1,3,4,5,9\}$ y usando (15), (17) y num $(Q(11))=5$, tenemos

$$
S^{1}=\left[\begin{array}{lllllllllll}
0 & 1 & 0 & 1 & 1 & 1 & 0 & 0 & 0 & 1 & 0 \\
0 & 0 & 1 & 0 & 1 & 1 & 1 & 0 & 0 & 0 & 1 \\
1 & 0 & 0 & 1 & 0 & 1 & 1 & 1 & 0 & 0 & 0 \\
0 & 1 & 0 & 0 & 1 & 0 & 1 & 1 & 1 & 0 & 0 \\
0 & 0 & 1 & 0 & 0 & 1 & 0 & 1 & 1 & 1 & 0 \\
0 & 0 & 0 & 1 & 0 & 0 & 1 & 0 & 1 & 1 & 1 \\
1 & 0 & 0 & 0 & 1 & 0 & 0 & 1 & 0 & 1 & 1 \\
1 & 1 & 0 & 0 & 0 & 1 & 0 & 0 & 1 & 0 & 1 \\
1 & 1 & 1 & 0 & 0 & 0 & 1 & 0 & 0 & 1 & 0 \\
0 & 1 & 1 & 1 & 0 & 0 & 0 & 1 & 0 & 0 & 1 \\
1 & 0 & 1 & 1 & 1 & 0 & 0 & 0 & 1 & 0 & 0
\end{array}\right], S^{0}=\left[\begin{array}{lllllllllll}
1 & 1 & 1 & 1 & 1 & 0 & 0 & 0 & 0 & 0 & 0 \\
1 & 1 & 1 & 1 & 1 & 0 & 0 & 0 & 0 & 0 & 0 \\
1 & 1 & 1 & 1 & 1 & 0 & 0 & 0 & 0 & 0 & 0 \\
1 & 1 & 1 & 1 & 1 & 0 & 0 & 0 & 0 & 0 & 0 \\
1 & 1 & 1 & 1 & 1 & 0 & 0 & 0 & 0 & 0 & 0 \\
1 & 1 & 1 & 1 & 1 & 0 & 0 & 0 & 0 & 0 & 0 \\
1 & 1 & 1 & 1 & 1 & 0 & 0 & 0 & 0 & 0 & 0 \\
1 & 1 & 1 & 1 & 1 & 0 & 0 & 0 & 0 & 0 & 0 \\
1 & 1 & 1 & 1 & 1 & 0 & 0 & 0 & 0 & 0 & 0 \\
1 & 1 & 1 & 1 & 1 & 0 & 0 & 0 & 0 & 0 & 0 \\
1 & 1 & 1 & 1 & 1 & 0 & 0 & 0 & 0 & 0 & 0
\end{array}\right]
$$

Aunque el método ofrece una diferencia relativa óptima y la menor expansión, este método tiene ciertas limitaciones, tales como el hecho de que el número de participantes tiene que ser un número primo, la imagen revelada siempre tiene distorsión en el aspecto de relación ancho y alto.

Las matrices básicas más sencillas para el esquema $(2,2)-C V$ son de tamaño $2 \times 2$ dado por

$$
S^{0}=\left[\begin{array}{ll}
1 & 0 \\
1 & 0
\end{array}\right] \quad, S^{1}=\left[\begin{array}{ll}
1 & 0 \\
0 & 1
\end{array}\right]
$$

La Fig. 9(a) muestra un ejemplo de un esquema (2,2)-CV usando las matrices básicas dadas por (19). Otras matrices básicas para (2,2)-CV pueden ser de tamaño $2 \times 4$ como 


$$
S^{0}=\left[\begin{array}{llll}
1 & 0 & 0 & 0 \\
1 & 0 & 0 & 0
\end{array}\right] \quad, S^{1}=\left[\begin{array}{llll}
1 & 0 & 0 & 0 \\
0 & 1 & 0 & 0
\end{array}\right]
$$

La Fig. 9(b) muestra un ejemplo de (2,2)-CV con $m=4$, usando las matrices básicas dadas por (20). De manera similar, para realizar un $(2,2)-C V$ con $m=4$, las siguientes matrices básicas también se pueden usar y la Fig. 9(c) muestra su realización.

$$
S^{0}=\left[\begin{array}{llll}
1 & 1 & 0 & 0 \\
1 & 1 & 0 & 0
\end{array}\right] \quad, S^{1}=\left[\begin{array}{llll}
1 & 1 & 0 & 0 \\
0 & 0 & 1 & 1
\end{array}\right]
$$

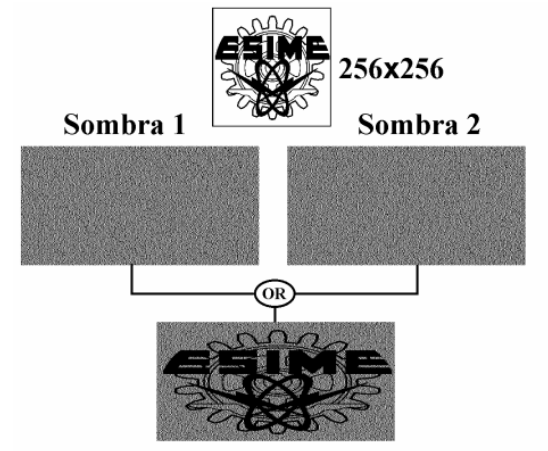

(a)

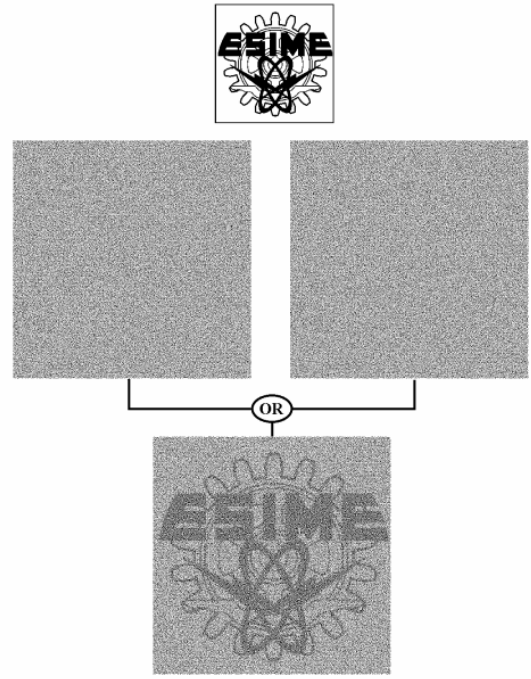

(b)

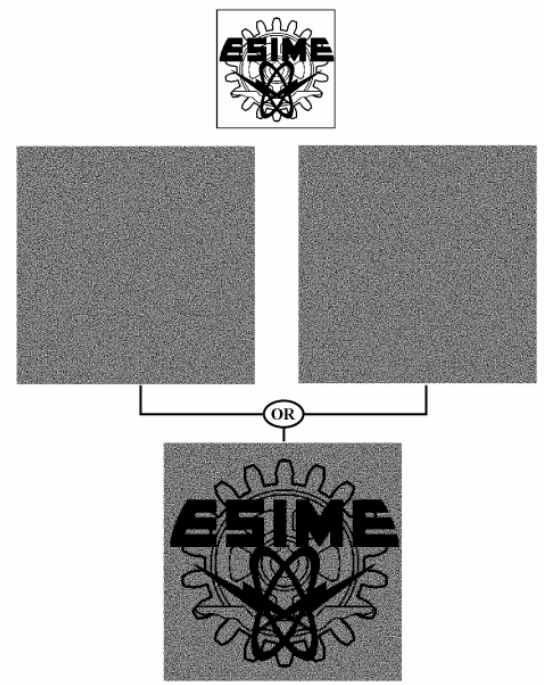

(c)

Fig. 9 Realización de (2,2)-VC . (a) usando matrices básicas (19) con $m=2$, (b) usando matrices básicas (20), con $m=4$, (c) usando matrices básicas (21).

En la realización de dos (2,2)-CV con $m=4$, la diferencia relativa a es $1 / 4$ y $1 / 2$, respectivamente, por lo tanto la realización usando las matrices básicas (21) es mejor, como se puede observar en la Fig. 9(c).

De la misma forma que en la $(2,2)-C V$, fácilmente se pueden construir las matrices básicas para $(2,3)-C V$ usando (8) y (9). En $(2,3)-C V$, el tamaño de las matrices básicas es de $3 \times m$, donde $m$ es factor de expansión. Dado que el factor de expansión óptimo es $m=3$, las matrices básicas están dadas por:

$$
S^{0}=\left[\begin{array}{lll}
1 & 0 & 0 \\
1 & 0 & 0 \\
1 & 0 & 0
\end{array}\right], \quad S^{1}=\left[\begin{array}{lll}
1 & 0 & 0 \\
0 & 1 & 0 \\
0 & 0 & 1
\end{array}\right]
$$

También las siguientes matrices básicas cumplen las condiciones de seguridad y contraste.

$$
S^{0}=\left[\begin{array}{lll}
1 & 1 & 0 \\
1 & 1 & 0 \\
1 & 1 & 0
\end{array}\right], \quad S^{1}=\left[\begin{array}{lll}
1 & 1 & 0 \\
0 & 1 & 1 \\
1 & 0 & 1
\end{array}\right]
$$

La Fig. 10 muestra las realizaciones de (2,3)-CV usando (22) y (23). 


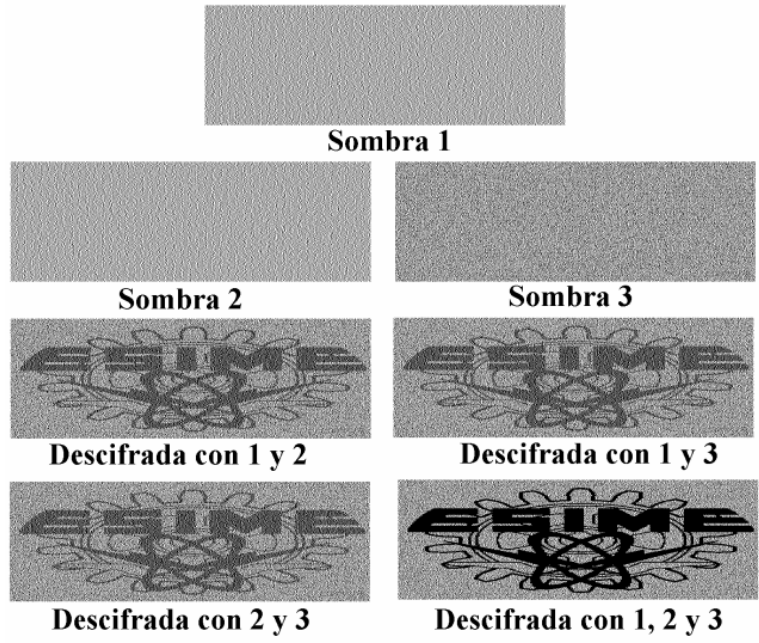

(a)

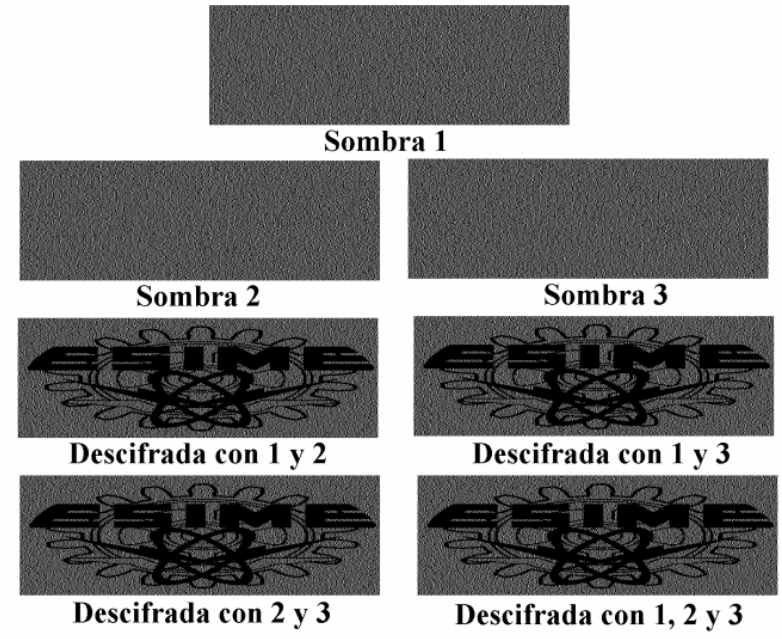

(b)

Fig. 10 Realización de (2,3)-CV usando (a) matrices básicas (22) y (b) matrices básicas (23).

En ambas realizaciones, la diferencia relativa $\alpha$ es 1/3. Las matrices básicas dadas en (24), desde el punto de vista del contraste cumplen la condición e inclusive mejor que (22) y (23), ya que la diferencia relativa $\alpha$ es $2 / 3$, sin embargo estas no cumplen la condición de seguridad, debido a que una sola sombra puede revelar el secreto.

$$
S^{0}=\left[\begin{array}{lll}
1 & 0 & 0 \\
1 & 0 & 0 \\
1 & 0 & 0
\end{array}\right], \quad S^{1}=\left[\begin{array}{lll}
1 & 1 & 0 \\
0 & 1 & 1 \\
1 & 0 & 1
\end{array}\right]
$$

Por ejemplo, considere la parte de una sombra la cual se muestra en la Fig. 11(a). Conociendo el factor de expansión (en este caso $m=3$ ) y observando el primer pixel contiene tres sub-pixeles de los cuales solo uno de ellos es igual a ' 1 ', mientras que el segundo pixel contiene tres sub-pixeles de los cuales dos de ellos son '1's. Si se asigna a cada pixel el color 'negro' o 'blanco' dependiendo del número de sub-pixeles con valor ' 1 ', se puede obtener fácilmente la imagen secreta como la mostrada en la Fig. 11(b).

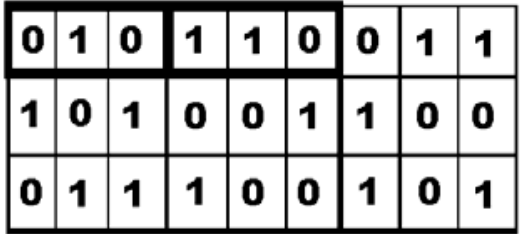

(a)

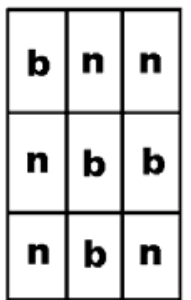

(b)

Fig. 11 (a) Ejemplo de una sombra generada por (24), (b) la imagen secreta revelada usando solamente la sombra (a).

Aquí 'b' y ' $n$ ' significan blanco y negro, respectivamente. Por lo tanto usando la sombra de un solo participante se puede revelar el secreto, lo cual violará la condición de seguridad.

Esquema (3,n)-CV

En el esquema $(3, n)-C V$, para revelar el secreto se requieren las sombras de 3 personas, las cuales se generan a través de las matrices básicas siguientes. 


$$
S^{1}=B \circ I, \quad S^{0}=\overline{S^{1}}
$$

donde $B$ es una matriz de tamaño $n x(n-2)$, cuyos elementos son unos e $I$ es la matriz identidad de $n x n$, la operación o denota la concatenación y la notación $\bar{X}$ es el complemento de la matriz $X$. Como un ejemplo, se construye el esquema $(3,4)$-CV usando (25) con $n=4$, entonces

$$
B=\left[\begin{array}{ll}
1 & 1 \\
1 & 1 \\
1 & 1 \\
1 & 1
\end{array}\right], \quad B \circ I=S^{1}=\left[\begin{array}{llllll}
1 & 1 & 1 & 0 & 0 & 0 \\
1 & 1 & 0 & 1 & 0 & 0 \\
1 & 1 & 0 & 0 & 1 & 0 \\
1 & 1 & 0 & 0 & 0 & 1
\end{array}\right], \quad S^{0}=\overline{S^{1}}=\left[\begin{array}{llllll}
0 & 0 & 0 & 1 & 1 & 1 \\
0 & 0 & 1 & 0 & 1 & 1 \\
0 & 0 & 1 & 1 & 0 & 1 \\
0 & 0 & 1 & 1 & 1 & 0
\end{array}\right]
$$

La Fig. 12 muestra realización de (3,4)-CV usando las matrices básicas (26). En el esquema (3,4)$\mathrm{CV}$, obviamente cualquier combinación de participantes cuyo número es menor que 3 , no puede revelar el secreto. La Fig. 12 también muestra resultados de la superposición de las sombras de algunas combinaciones de los participantes no calificados. El factor de expansión del esquema mostrado es $m=6$, por lo tanto la razón de aspecto ancho y alto de la imagen revelada fue modificada en comparación a la original. La diferencia relativa (contraste relativo) es de 1/6.

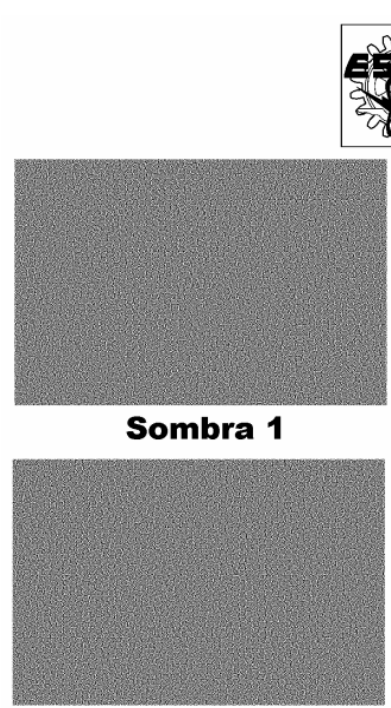

Sombra 3

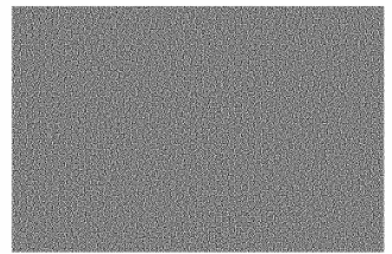

Sombra 2

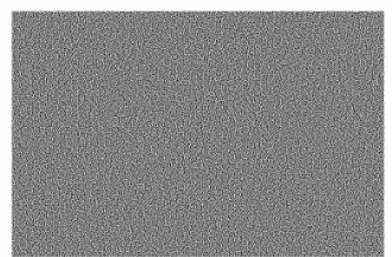

Sombra 4

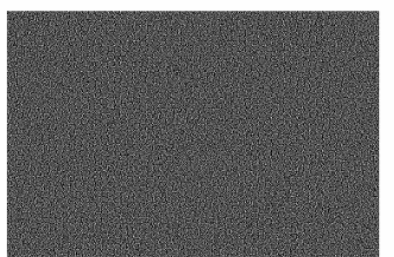

Superpuestos dos sombras

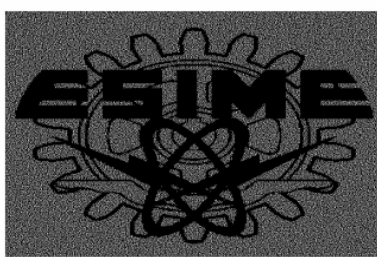

Superpuestos cuatro sombras

Fig.12 Realización del esquema (3,4)-CV. (a) sombras generadas y resultados de las superposiciones.

\section{Esquema $(n, n)-C V$}

En este esquema, para revelar el secreto se requieren las sombras de todos los participantes. En este caso el método de construcción de las matrices básicas se describe a continuación. Inicialmente en el esquema $(n, n)-\mathrm{CV}$, el factor de expansión es siempre $m=2^{n-1}$, por lo tanto el número de columnas de las matrices básicas es $m=2^{n-1}$ y el número de renglones es $n$. Así la condición que deben cumplir $S^{0}, S^{1}$ es la siguiente: En cada columna de $S^{0}$, el número de '1' es par, mientras que en cada columna de $S^{1}$, el número de 1 es impar.

Así, las matrices básicas del esquema (3,3)-CV están dadas por 


$$
S^{0}=\left[\begin{array}{llll}
0 & 1 & 0 & 1 \\
0 & 1 & 1 & 0 \\
0 & 0 & 1 & 1
\end{array}\right] \quad, \quad S^{1}=\left[\begin{array}{llll}
1 & 0 & 0 & 1 \\
0 & 1 & 0 & 1 \\
0 & 0 & 1 & 1
\end{array}\right]
$$

Mientras que las matrices básicas del $(4,4)$-CV son

$$
S^{0}=\left[\begin{array}{llllllll}
0 & 1 & 1 & 0 & 0 & 0 & 1 & 1 \\
0 & 1 & 0 & 1 & 0 & 1 & 0 & 1 \\
0 & 0 & 1 & 0 & 1 & 1 & 0 & 1 \\
0 & 0 & 0 & 1 & 1 & 0 & 1 & 1
\end{array}\right] \quad, \quad S^{1}=\left[\begin{array}{llllllll}
1 & 0 & 0 & 0 & 1 & 0 & 1 & 1 \\
0 & 1 & 0 & 0 & 1 & 1 & 0 & 1 \\
0 & 0 & 1 & 0 & 1 & 1 & 1 & 0 \\
0 & 0 & 0 & 1 & 0 & 1 & 1 & 1
\end{array}\right]
$$

La Fig. 13 muestran la realización de $(3,3)-C V$ y $(4,4)-C V$ usando las matrices básicas dadas por (27) y (28).

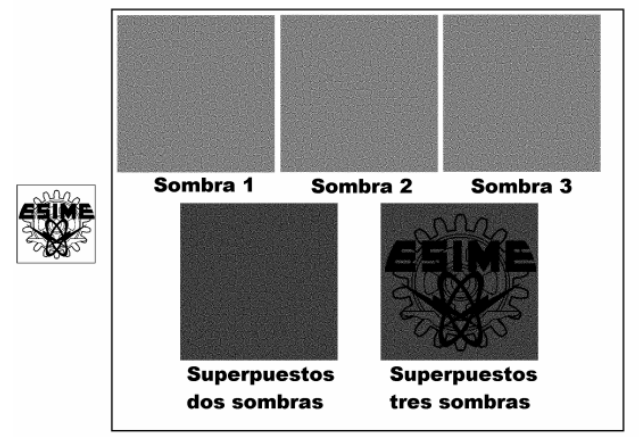

(a)

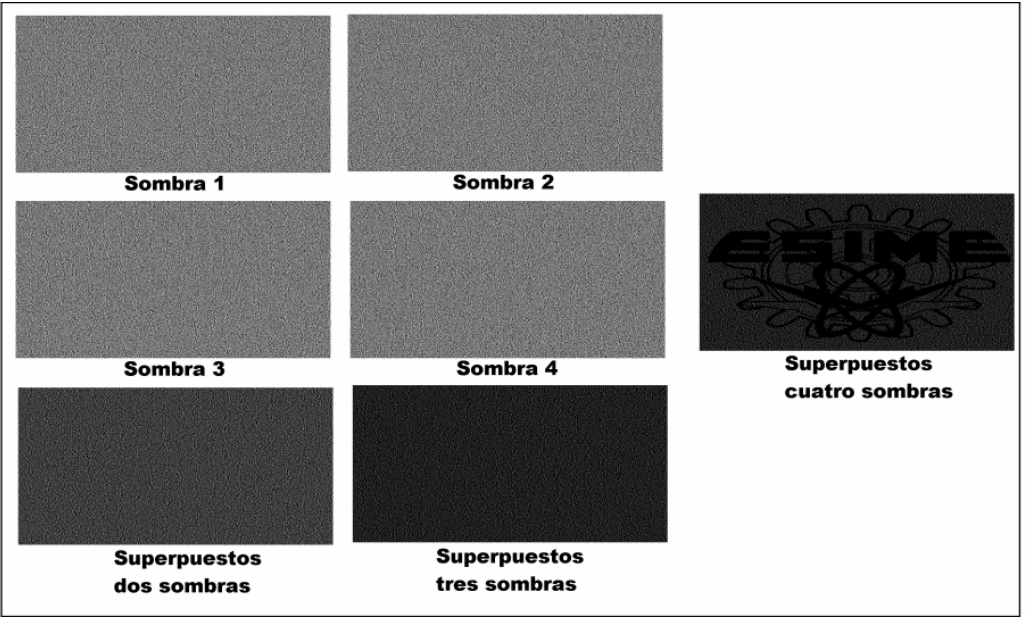

(b)

Fig. 13 Realización del esquema (n,n)-CV, (a) (3,3)-CV, (b) (4,4)-CV.

En ambas figuras, se presentan solo algunas combinaciones de participantes, por cuestión de espacio.

\section{CONCLUSIONES}

Este artículo presentó una revisión tutorial sobre criptografía visual (CV) basada en el esquema de umbral $(k, n)$-CV propuesto por Naor y Shamir, junto con una serie detallada de ejemplos que ilustran los conceptos analizados. En la sección de conceptos básicos de CV del artículo, se introdujeron asuntos comunes entre algunas variantes existentes de $\mathrm{CV}$, los cuales son términos básicos, algoritmo genérico de cifrado y descifrado en $\mathrm{CV}$, y aspecto de seguridad y contraste de CV. La discusión presentada muestra que los esquemas $(k, n)-C V$, cuando $k=2$, presentan factores de expansión menores que los esquemas de $C V$ con $k>2$, aunque estos son menos seguros ya que para recuperar la imagen secreta se requieren solamente dos participantes, por otro lado conforme k se incrementa el sistema adquiere mayor seguridad ya que un mayor número de participantes son necesarios para recuperar la imagen secreta, aunque en estas situaciones el factor de expansión se incrementa. Relativo a la complejidad computacional, todos los esquemas analizados muestran una complejidad computacional similar ya que, en todos los casos, el método para generar las sombras es el mismo y en todos los casos las imágenes se recuperan o descifran superponiendo las sombras. Finalmente, para que los sistemas de CV alcancen su madurez y sean empleados en la solución de problemas prácticos, asuntos de contraste y el factor de expansión deben ser discutidos y optimizados. 


\section{AGRADECIMIENTOS}

Los autores agradecen al Instituto Politécnico Nacional de México, a la Universidad de ElectroComunicaciones de Japón y al Consejo Nacional de Ciencia y Tecnología de México por el apoyo económico proporcionado durante la realización de este trabajo.

\section{REFERENCIAS}

Ateniese, G., C. Blundo, A. De Santis y D. R. Stinson, Visual Cryptography for General Access Structures, Inform. Comput, 129(2), 86-106 (1996).

Ateniese, G., C. Blundo, A. De Santis y D. R. Stinson, Extended Capability for Visual Cryptography, Theoretical Computer Science, 250(1-2), 143-161 (2001).

Blundo, C., A. De Santis y D. R. Stinson, On the Contrast in Visual Cryptography Schemes, Journal of Cryptography 12(1), 261-289 (1999).

Blundo A., A. De Bonis y A. De Santis, Improved Schemes for Visual Cryptography, Designs, Codes and Cryptography, Kluwer Academic Publishers, 24, 255-278 (2001).

Blundo C., S. Cimato y A. De Santis, Visual Cryptography Schemes with Optimal Pixel Expansion, Theoretical Computer Science, Elsevier, 369, 169-182 (2006).

Droste, S., New Results on Visual Cryptography, Lecture Notes in Computer Science, Advances in Cryptology, LNCS 1109, Springer-Verlag, 401-415 (1996).

Hajiabolhassan H. y A. Cheraghi, "Bounds for Visual Cryptography Schemes", Discrete Applied Mathematics, Elsevier, 158, 659-665 (2010).

Hegde C., S. Manus, P. D. Shenoy, K. R. Vengopal y L. M. Patnaki, Secure Authentication using Image Processing and Visual Cryptography for Banking Applications, $16^{\text {th }}$ Int. Conf. on Advanced Comp. and Comm, 65-72 (2008).

Klein A. y M. Wessler, Extended Visual Cryptography Schemes, Inform. Compt. 205(5), 716-732 (2007).

Koga H., A General Formula of the (t,n)-Threshold Visual secret sharing scheme, Advances in Cryptology-Asiacrypt 2002. LNCS 2001, 328-345 (2002).

Mese, M. y P. Vaidyanathan, Recent Advances in Digital Halftoning and Inverse Halftoning Methods, IEEE Trans. on Circuits and Systems-I, 49(6), 790-805 (2002).

Mora F. J. y Y. A. Torrabia, Nuevas Formas de Encripción para Archivos Multimedia. La encripción perceptiva, Revista de Información Tecnológica, 13(5), 91-96 (2002).

Naor M. y A. Shamir, Visual Cryptography, Advances in Cryptography-Eurocrypt'94, 1-12 (1994).

Naor M. y A. Shamir, Visual Cryptography II: Improving the contrast via the cover base, Lecture Note in Computer Science, 1189, 197-202 (1997).

Rao, Y., Y. Sukonkina, C. Bhagwati y U. K. Singh, Fingerprint based authentication application using visual cryptography methods, 2008 IEEE Region 10 Conf.,1-5 (2008).

Shamir A., How to Share a Secret, Communications of the ACM, 22(10), 612-613 (1979).

Stinson D., Visual Cryptography and threshold schemes, IEEE Potential, 13-16 (1999).

Verheul E., C. A. Henk y V. Tilborg, Constructions and Properties of $k$ out of $n$ Visual Secret Sharing Schemes, Designs, Codes and Cryptography, Kluwer Academic Publishers, 11, 179-196 (1997). 